\title{
VEGF induces angiogenesis in a zebrafish embryo glioma model established by transplantation of human glioma cells
}

\author{
DONG LI ${ }^{2}$, XIANG-PEN LI ${ }^{1}$, HONG-XUAN WANG ${ }^{1}$, QING-YU SHEN ${ }^{1}$, XIANG-PING LI ${ }^{3}$, \\ LU WEN $^{2}$, XIU-JIAO QIN ${ }^{2}$, QIU-LI JIA ${ }^{2}$, HSIANG-FU KUNG ${ }^{2,4,5}$ and YING PENG ${ }^{1,2}$ \\ ${ }^{1}$ Department of Neurology, Sun Yat-Sen Memorial Hospital, Sun Yat-Sen University, Guangzhou 510120; \\ ${ }^{2}$ Laboratory of Integrated Biosciences, School of Life Sciences, Sun Yat-Sen University, Guangzhou 510006; \\ ${ }^{3}$ Department of ENT, Nanfang Hospital, Southern Medical University, Guangzhou 510515; \\ ${ }^{4}$ Stanley Ho Center for Emerging Infectious Diseases, and Li Ka Shing Institute of Health Sciences, \\ The Chinese University of Hong Kong, Hong Kong; ${ }^{5}$ State Key Laboratory of Oncology \\ in Southern China, Cancer Center, Sun Yat-Sen University, Guangzhou, P.R. China
}

Received March 6, 2012; Accepted May 8, 2012

DOI: $10.3892 / o r .2012 .1861$

\begin{abstract}
Zebrafish (Danio rerio) is becoming an increasingly popular vertebrate cancer model. In this study, we established a xenotransplanted zebrafish embryo glioma model to further investigate the molecular mechanisms of tumor angiogenesis. We find that the glioma cell line U87 can survive, proliferate and induce additional SIV branches in zebrafish embryos. In addition, by the means of in situ hybridization and quantitive RT-PCR analyses we find that the transplanted U87 cells can induce the ectopic zebrafish vascular endothelial growth factor A (VEGF A) and its receptor VEGFR2/KDR mRNA expression and increase their expression levels, resulting in additional SIV branches.
\end{abstract}

\section{Introduction}

Glioma is the most common primary brain tumor in adults and the median survival for patients without therapy is up to 3 months (1). Currently the traditional treatment such as resection, chemotherapy and radiotherapy has not greatly improved the median survival for patients. Malignant glioma is notorious for its behavior of rapid proliferation, great invasion and

Correspondence to: Dr Ying Peng, Department of Neurology, Sun Yat-Sen Memorial Hospital, Sun Yat-Sen University, No. 107 West Yanjiang Road, Guangzhou, P.R. China

E-mail: docpengy@yahoo.com.cn

Abbreviations: hpf, hour post-fertilization; dpf, day post-fertilization; hpi, hour post-injection; dpi, day post-injection; DMEM, Dulbecco's modified Eagle's medium; SIV, subintestinal vessel; PTU, 1-phenyl2-thiourea; PBS, phosphate buffered saline; PFA, paraformaldehyde; qPCR, quantitative real-time PCR

Key words: zebrafish, glioma model, angiogenesis, VEGF, KDR metastasis and it is also among the best vascularized tumors in humans. It is well known that angiogenesis plays a critical role in tumor progression. Therefore new anti-angiogenic treatment strategies are required (2-6). Revealing of various signaling pathways that lead to activation of angiogenesis will provide molecular insight into developing therapeutic agents to treat glioma. In the past decades, researchers have discovered many molecular mechanisms related to tumor angiogenesis (7-9).

Traditional rodent cancer models have disadvantages, e.g., the high cost, long developmental phase, and limited availability for high-throughput assays (10), which limited effective ways to find the new molecular mechanisms and monitor the tumor in vivo in real time.

The teleost zebrafish (Danio rerio), a promising alternative vertebrate cancer model has attracted considerable attention in recent years because of compelling advantages of transparency of the embryo, rapid development, fecundity, tractable genetics, great screening efficiency, high levels of physiologic and genetic homology with higher vertebrates (11-13). Moreover, the zebrafish embryo within 1 week is immune-free, and showed no immunosuppression to transplanted human glioma cells (14). The transgenic zebrafish strains, e.g., casper transparent mutant line (15), and VEGFR2:G-RCFP line with green fluorescence specifically in blood vessels (16), offered new tools for cancer research.

In this study, we microinjected human U87 glioma cells into zebrafish embryos and developed a xenograft zebrafish glioma model. The U87 cells are first labeled with a red fluorescence protein and then microinjected into perivitelline space of VEGFR2:G-RCFP transgenic zebrafish embryos at $48 \mathrm{~h}$ post-fertilization. Then in order to investigate the angiogenesis mechanisms in details, we make use of the staining of endogenous alkaline phosphatase, fluorescent microscopy monitoring, in situ hybridization and quantitive RT-PCR to investigate the change of glioma related molecules in a zebrafish embryo angiogenesis glioma model. 


\section{Materials and methods}

Glioma cell culture and transfection. The human malignant glioma cell line U87 (American Type Culture Collection) was maintained in 90\% DMEM containing $100 \mathrm{U} / \mathrm{ml}$ penicillin, $100 \mu \mathrm{g} / \mathrm{ml}$ streptomycin (Gibco, NY, USA) and $10 \%$ fetal bovine serum (Invitrogen, $\mathrm{CA}$, USA) with $5 \% \mathrm{CO}_{2}$ at $37^{\circ} \mathrm{C}$. Plasmid DNA labeled with red fluorescent protein DNA fragment (pcDNA3.0-DsRed) was provided by Dr Ben-Ping Luo and is amplified using Endo-Free Plasmid Mini kit I (Omega, USA). One day before transfection, we plated $0.5-2 \times 10^{5}$ U87 cells in $500 \mu \mathrm{l}$ of growth medium without antibiotics in each well of a 24 -well plate so that cells are $90-95 \%$ confluent at the time of transfection. Then these cells were transfected with pcDNA3.0-DsRed vector using Lipofectamine 2000 (Invitrogen) as per the instructions with slight modification. The U87 glioma cells expressing red fluorescent protein (U87-DsRed glioma cells) were selected using $300 \mu \mathrm{g} / \mathrm{ml}$ G418 and cultured for future use.

Zebrafish incubation. The adult AB zebrafish and VEGFR2:GRCFP (provided by integrated laboratory of Sun Yat-Sen University) transgenic zebrafish were maintained in zebrafish breeding system as previously described (17). The zebrafish embryos were incubated in petri dishes with $\mathrm{E} 3$ buffer $(5 \mathrm{mM}$ $\mathrm{NaCl}, 0.17 \mathrm{mM} \mathrm{KCl}, 0.33 \mathrm{mM} \mathrm{CaCl}_{2}, 0.33 \mathrm{mM} \mathrm{MgSO}_{4}$ ) at $28^{\circ} \mathrm{C}$ until they were ready for use. If the zebrafish embryos are used after $48 \mathrm{hpf}$, it should be placed in E3 buffer with 1-phenyl2-thiourea (PTU) (Sigma-Alrich, USA) at $24 \mathrm{hpf}$ and the buffer should be changed every day in order to eliminate melanin. The embryos containing chorion should be dechorioned with 1 $\mathrm{mg} / \mathrm{ml}$ pronase (Sigma-Alrich) and then anesthetized with $0.04 \mathrm{mg} / \mathrm{ml}$ tricaine (Sigma-Alrich) before they are microinjected with cells( 18).

The establishment of zebrafish embryo glioma model. Firstly, the prepared U87-DsRed glioma cells were trypsinized and suspended in Matrigel (R\&D, USA) at a density of $\sim 10^{8}$ cells/ $\mathrm{ml}$. Then Narishige microinjector was used to inject 10-30 nl suspended U87-DsRed cells into the perivitelline space near SIV of the VEGFR2:G-RCFP transgenic zebrafish embryos at 48 hpf $(18,19)$ to establish this zebrafish embryo glioma model. The blank matrigel injected zebrafish embryos were used as a negative control.

Fluorescent microscopy monitoring. When the zebrafish embryo glioma model was set up a Nikon epifluorescent microscope was used to monitor the progression of glioma cells and the subintestinal vessel (SIV) changes of transgenic VEGFR2RCFP zebrafish in real time continually for 2 days.

Whole-mount endogenous alkaline phosphatase staining. In order to further investigate the angiogenesis ability of glioma cells, we fixed the U87 injected zebrafish embryos at 1 day post-injection (dpi) and 2 dpi in phosphate buffered saline (PBS)-4\% paraformaldehyde (PFA) for $2 \mathrm{~h}$ at room temperature and then stained for endogenous alkaline phosphatase activity following the protocol of Serbedzija et al (20). Then embryos were observed and photographed under a Nikon stereomicroscope.
Whole-mount in situ hybridization. We further investigated the molecular mechanisms of glioma angiogenesis by in situ hybridization test of zebrafish VEGF A and VEGFR2/KDR mRNA expression in zebrafish embryo glioma model using VEGF A and KDR digoxigenin-labeled antisense RNA probes. At first, we cloned part of the zebrafish VEGF and KDR sequence which were $\sim 1 \mathrm{~kb}$ into the pcDNA3.0 plasmid. The primers were as follows: VEGF (forward: 5'-ttggaattcagcgactcaccgcaacactc-3', reverse: 5'-ataaagcttcattcgttgttccgctcctg-3'); KDR (forward: 5'-gcagaattcattcccatgccgaacattac-3', reverse: 5'-gttaagcttagtctg aggcgatcttgagg-3'). Then by using the DIG System nucleic acid labeling kit (Roche, USA), we prepared these two probes with $\mathrm{T} 7$ promoter in vitro and then observed the signals by using an NBT/BCIP staining solution (Roche). The details of the high-resolution in situ hybridization protocol have been reported (21). We used a blank Matrigel injected zebrafish as a negative control for zebrafish angiogenesis glioma model in whole-mount in situ hybridization. The experiments were repeated 3 times.

Quantitive RT-PCR analysis. To determine the exact change of zebrafish VEGF A and VEGFR2 mRNA expression in zebrafish embryo glioma model we also carried out quantitive RT-PCR tests of zebrafish VEGF and VEGFR2 mRNA expression. The qRT-PCR primers were as follows: VEGF (forward: 5'-tgctcctgcaaattcacacaa-3', reverse: 5'-atcttggcttttcacatctgcaa-3'); KDR (forward: 5'-tggagttccagcaccettta-3', reverse: 5 '-cgtccttcttcaccetttca-3'); $\beta$-actin (forward: 5 '-cgtgacatcaa ggagaagct-3', reverse: 5'-tcgtggataccgcaagattc-3'). Total RNA was extracted from 40 glioma angiogenesis zebrafish embryos and then treated with DNase I. Promega reverse transcription kit was used to get the zebrafish single-stranded cDNA. Then we used the SYBR GreenER ${ }^{\mathrm{TM}}$ qPCR SuperMix Universal kits (Applied Biosystems Inc.) to carry out quantitive PCR in Applied Biosystems 7500 Real-Time PCR system (Applied Biosystems Inc.) according to the manufacturer's instructions.

Data were normalized with zebrafish $\beta$-actin and repeated 3 times with similar results. Ct-value for each sample was calculated with the ${ }^{\Delta \Delta} \mathrm{Ct}$-method (22) and results were expressed as $2^{-\triangle \Delta C T}$. A blank Matrigel injected zebrafish was used as a negative control.

\section{Results}

Human glioma cells U87 can survive, proliferate and induce angiogenesis in zebrafish embryos. We established the zebrafish embryo glioma model and we monitored the activity of U87 cells and changes of SIV in zebrafish embryo glioma model by the use of epifluorescent microscopy. The human U87 glioma cells can survive, proliferate and induce angiogenesis in zebrafish embryos from 24 to 48 hpi (Fig. 1). The angiogenesis ability increased from 1 to 2 dpi (Fig. 1D and F). We calculated the percentage of additional SIV branches in the zebrafish embryo and also found that the percentage of positive SIV phenotype increased from 1 to 2 dpi (Fig. 2).

Endogenous alkaline phosphatase staining for zebrafish embryo glioma model showed similar results on additional SIV branches to that observed by the epifluorescent microscope (Fig. 3B and D). 

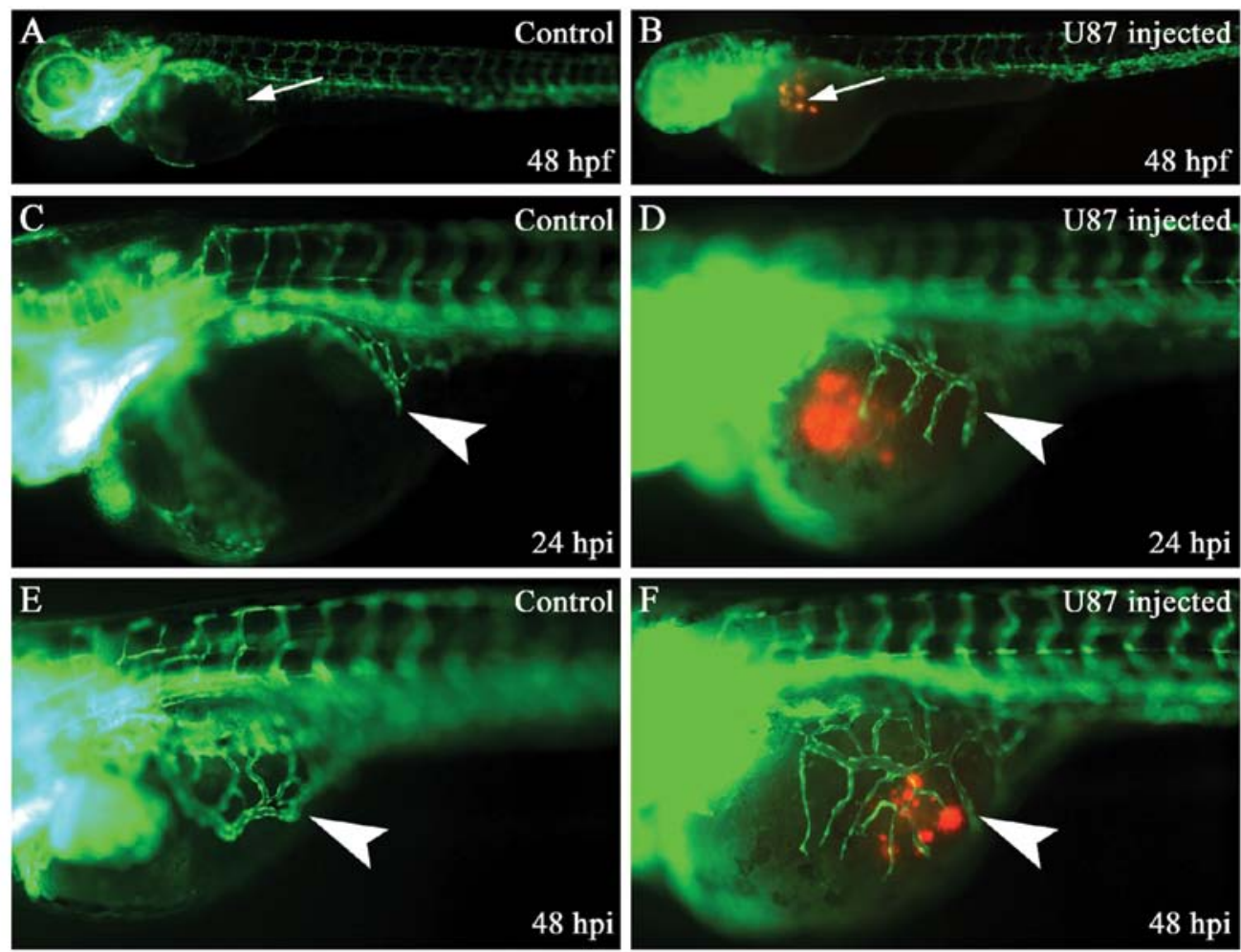

Figure 1. The U87 human glioma cells survive, proliferate and induce angiogenesis in zebrafish embryo glioma model. The SIV pattern in transgenic (tg) (VEGFR2:G-RCFP) embryos injected with matrigel as a negative control (A, C and E) and U87 cells (B, D and F) are shown under an epifluorescent microscope. The microinjection sites of $48 \mathrm{~h}$ pf embryos are shown by white arrow in control matrigel injected embryo (A) and U87 (red dots) injected embryo (B). The matrigel injected tg(VEGFR2:G-RCFP) embryo (C) and U87 (red dots) injected tg(VEGFR2:G-RCFP) embryo (D) showed green fluorescent SIVs at 24 hpi (lateral view, head on the left). The SIV (white arrowhead) developed normally in control matrigel injected embryos from 24 to 48 hpi (C and E). The SIV (white arrowhead) developed additional branches in U87 (red dots) injected embryos from 24 to 48 hpi (D and F). (A and B) Magnification x40; (C-F) Magnification x100.

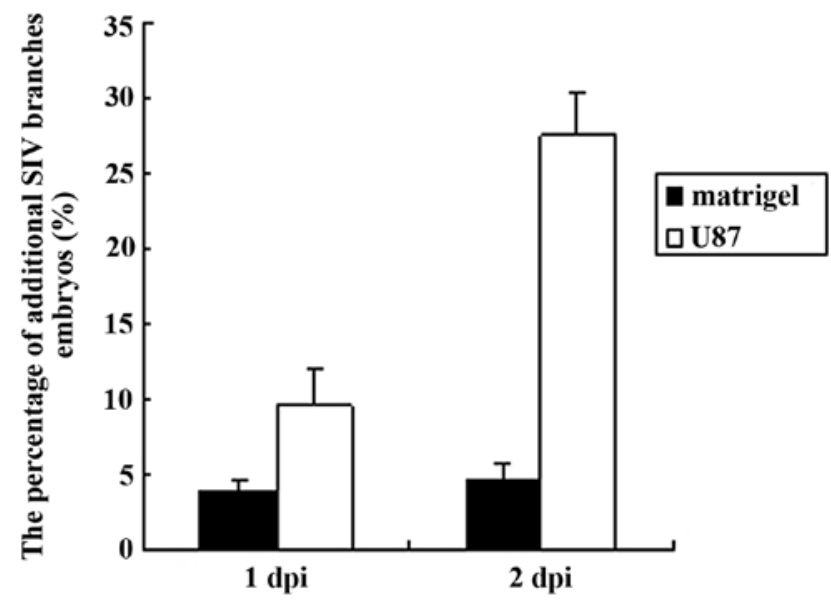

Figure 2. The percentage change of the additional SIV embryos in zebrafish embryo glioma model from 1 to 2 dpi. Data are expressed as percentage of embryos which have the additional SIV branches. The results are the mean \pm SD of three respective groups of U87 injected embryos (40 embryos/group) The Matrigel injected embryos were used as a negative control. The data are from 1 to 2 dpi.

Human glioma cells U87 can induce the ectopic zebrafish VEGF and VEGFR2 mRNA expression and increase their expression quantity. By in situ hybridization and quantitive RT-PCR test of zebrafish VEGF and VEGFR2 mRNA expression in zebrafish embryo glioma model from 1 to $2 \mathrm{dpi}$, we found that the expression area of VEGF and VEGFR2 mRNA around SIV had enlarged (Fig. 4) and their expression was also increased (Fig. 5).

\section{Discussion}

The zebrafish glioma model provides a convenient in vivo system for the study of cancer cell molecular mechanisms. Many other kinds of zebrafish cancer models have already appeared worldwide. These models mainly include three different types. The first type is that Spitsbergen et al $(23,24)$ gained by chemical induction and acquiring several types of cancers in zebrafish through chemical carcinogenesis such as epithelial, mesenchymal, neural neoplasia induced by 7,12-dimethylbenz[a]anthracene and hepatic, mesenchymal neoplasia induced by N-methyl-N'-nitro-N-nitrosoguanidine. The second type of zebrafish cancer model is obtained by genetic manipulation, bmyb mutation causes genome instability and increased cancer susceptibility in zebrafish (25). The third transplanted zebrafish glioma model is that we described herein. Amatruda et al have also reported a study in Cancer Cell (26). A zebrafish cancer model was also presented July 2009 in Spoleto, Italy, recapitulating a number of new zebrafish cancer models (27), indicating that zebrafish model could make a great contribution to cancer research. 

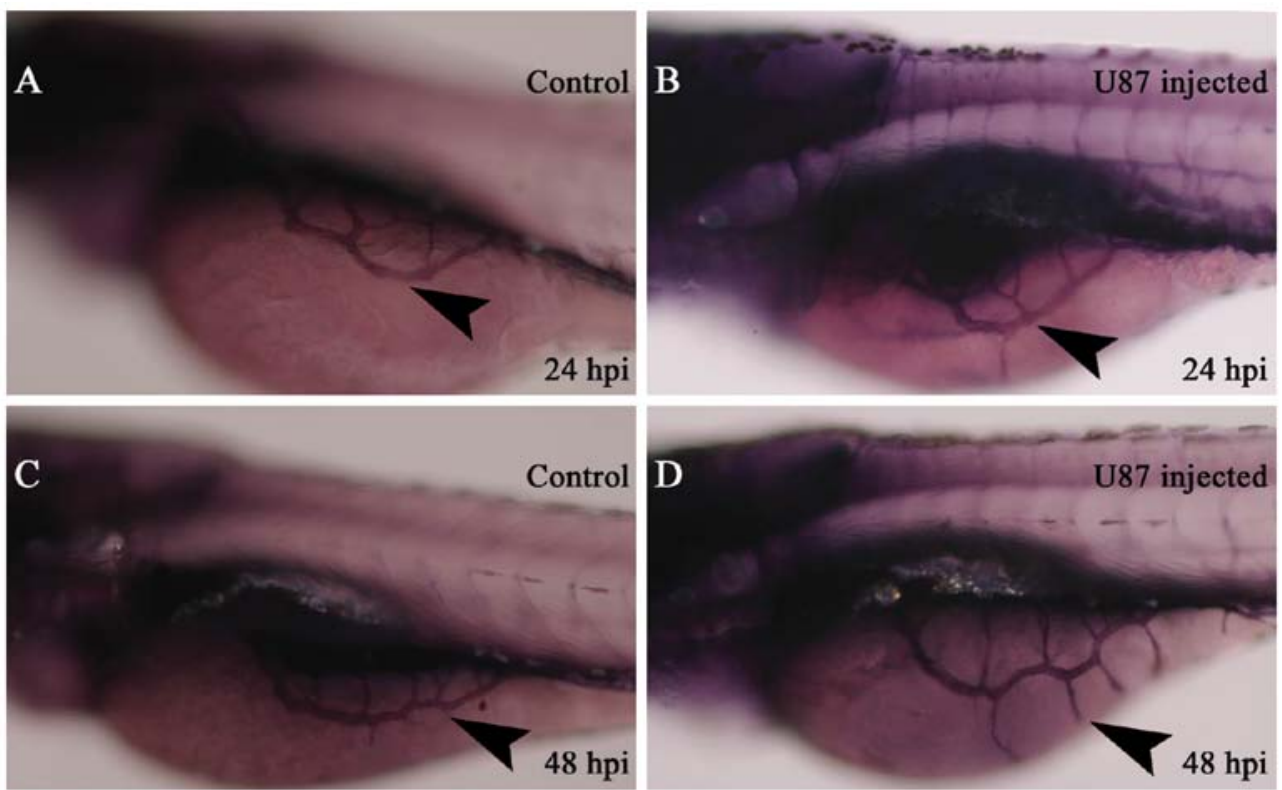

Figure 3. Whole-mount endogenous alkaline phosphatase staining of a zebrafish embryo glioma model. Matrigel injected embryos (control) are shown in (A) (24 hpi) and (C) (48 hpi). U87 injected embryos are shown in (B) (24 hpi) and (D) (48 hpi). The blood vessels are stained purple. The SIV is shown by black arrowhead. All zebrafish are shown with head on the left. Magnification x100.
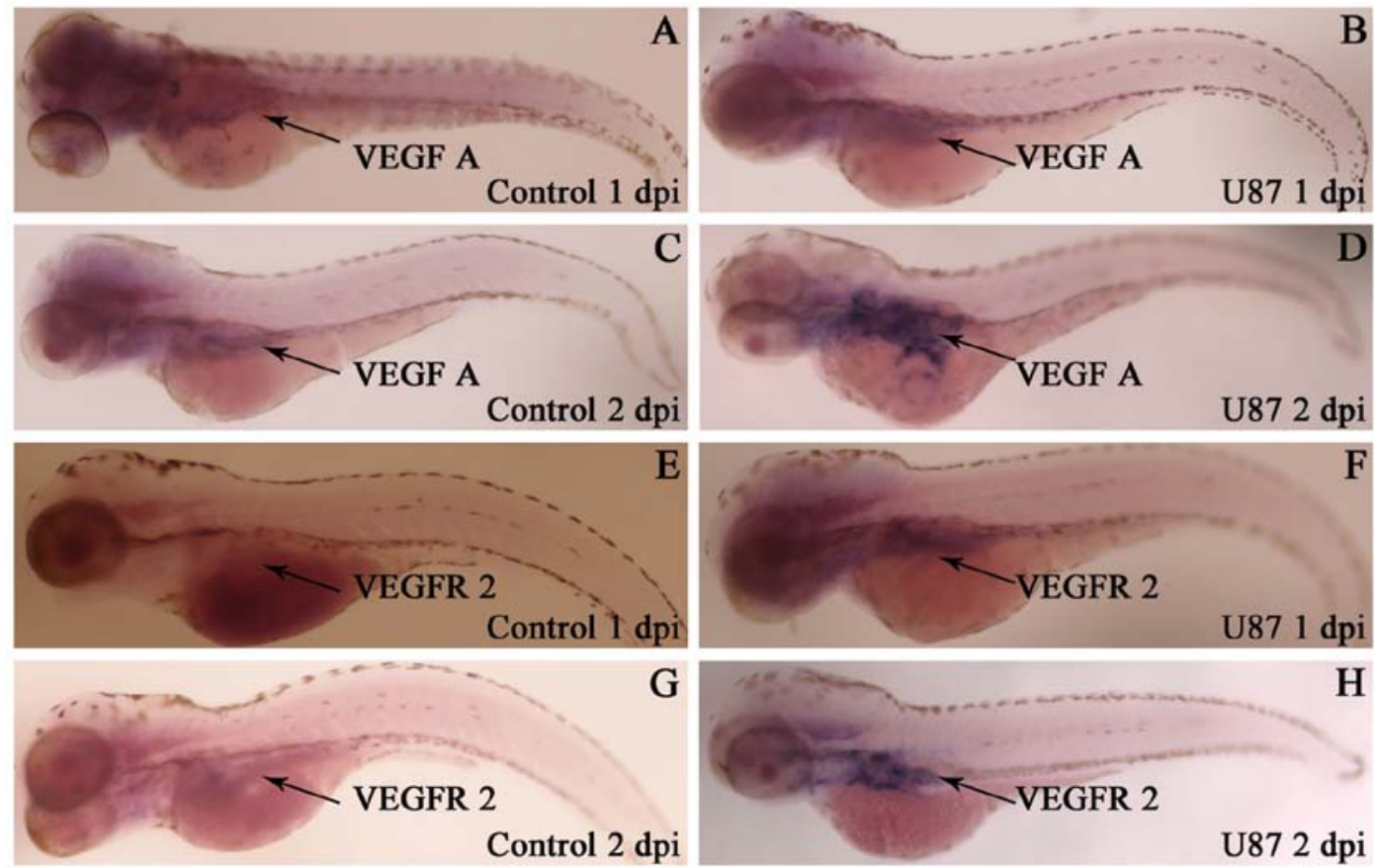

Figure 4. In situ hybridization of VEGF and VEFGR2 mRNA expression in a zebrafish embryo glioma model. The results are shown in matrigel injected control embryos (A, C, E and G) and U87 injected embryos (B, D, F and H). The VEGF A signals are shown by black arrow in control from 1 (A) to 2 dpi (C) and in U87 injected embryos from 1 (B) to 2 dpi (D). The VEGFR2 signals are shown by black arrow in control from 1 (E) to 2 (G) and in U87 injected embryos from 1 (F) to $2 \mathrm{dpi}(\mathrm{H})$. All the embryos are shown with head on the left and $\mathrm{x} 40$ images under a Nikon stereomicroscope.

Glioma is a notorious malignant cancer in humans and research has been done into its molecular mechanisms in vitro and in vivo for many years. In this study, we found that the U87 human glioma cells can survive, proliferate and induce angiogenesis in a zebrafish embryo. As is known, angiogenesis is a key factor in malignant tumor progression (28) and here these transplanted glioma cells U87 can also induce additional subintestinal vessels and transfer to the distant site through intravasation and extravasation.

Vascular endothelial growth factors (VEGFs) play an important part in angiogenesis $(29,30)$ and currently include VEGF-A,-B,-C,-D,-E and placenta growth factor (PIGF) in its family. They play a crucial role in the process of angiogenesis by binding tyrosine kinase receptors such as VEGF receptor-1, 


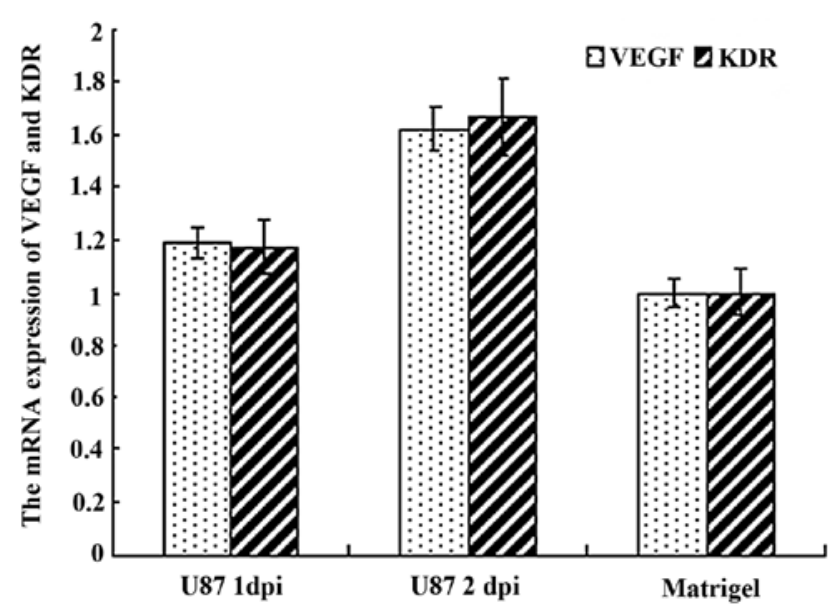

Figure 5. The mRNA expression level of VEGF and VEGFR2/KDR revealed by qPCR in a zebrafish embryo glioma model. The relative level of VEGF and VEGFR2 in negative control (matrigel injected embryos) was normalized to 1, and the Ct values of VEGF and VEGFR2 in zebrafish embryo glioma model from 1 to $2 \mathrm{dpi}$ were used to represent the relative level of VEGF and VEGFR2. Results are expressed as $2^{-\Delta \Delta C T}\left(n=3 ;{ }^{*} p<0.05\right)$.

-2 , and -3 (31). We carried out an investigation on VEGF A and its receptor VEGFR2/KDR $(32,33)$, we found that the transplanted human U87 glioma cells can not only increase the mRNA expression of zebrafish VEGF A and VEGFR2 but also induce the secretion of VEGF $A$ and VEGFR2 mRNA in different anatomic sites in zebrafish, and these molecules contribute to induction of additional zebrafish SIV branches. This result is consistent with previous published reports. Serbedzija et al have reported that VEGF can induce angiogenesis by the injection of its protein directly into zebrafish embryo (20) and Habeck et al have also shown a very similar result to ours by injecting VEGF plasmid into zebrafish embryo (34). The above evidence proves that VEGF indeed plays an important role in zebrafish embryo angiogenesis. The injected human glioma cells U87 induced zebrafish embryo angiogenesis further confirms that zebrafish angiogenesis-related genes show great conservation with humans, as Liang et al (35) previously showed that zebrafish VEGF's functional sites.

\section{Acknowledgements}

We thank Benping Luo for providing the vector pcDNA3.0DsRed. This study was supported by the National Natural Science Foundation of China (30973479 to Y.Peng), the National High Technology Research and Development Program of China (863 Program) (2007AA021101 to Y. Peng), and Science and Technology Planning Project of Guangdong Province, China (2009B060700040 and 2011B031800141 to Y. Peng).

\section{References}

1. Ng SS, Gao Y, Chau DH, et al: A novel glioblastoma cancer gene therapy using AAV-mediated long-term expression of human TERT C-terminal polypeptide. Cancer Gene Ther 14: 561-572, 2007.

2. Lin ZX, Yang LJ, Huang Q, et al: Inhibition of tumor-induced edema by antisense VEGF is mediated by suppressive vesiculovacuolar organelles (VVO) formation. Cancer Sci 99: 2540-2546, 2008 .
3. Paez-Ribes M, Allen E, Hudock J, et al: Antiangiogenic therapy elicits malignant progression of tumors to increased local invasion and distant metastasis. Cancer Cell 15: 220-231, 2009.

4. Norden AD, Drappatz J and Wen PY: Novel anti-angiogenic therapies for malignant gliomas. Lancet Neurol 7: 1152-1160, 2008.

5. Vredenburgh JJ, Desjardins A, Herndon JN, et al: Bevacizumab plus irinotecan in recurrent glioblastoma multiforme. J Clin Oncol 25: 4722-4729, 2007

6. Tuettenberg J, Friedel C and Vajkoczy P: Angiogenesis in malignant glioma - a target for antitumor therapy? Crit Rev Oncol Hematol 59: 181-193, 2006.

7. Gan HK, Lappas M, Cao DX, Cvrljevdic A, Scott AM and Johns TG: Targeting a unique EGFR epitope with monoclonal antibody 806 activates NF-kappaB and initiates tumour vascular normalization. J Cell Mol Med 13: 3993-4001, 2009.

8. Jang FF, Wei W and De WM: Vascular endothelial growth factor and basic fibroblast growth factor expression positively correlates with angiogenesis and peritumoural brain oedema in astrocytoma. J Ayub Med Coll Abbottabad 20: 105-109, 2008.

9. di Tomaso E, London N, Fuja D, et al: PDGF-C induces maturation of blood vessels in a model of glioblastoma and attenuates the response to anti-VEGF treatment. PLoS One 4: e5123, 2009.

10. Huse JT and Holland EC: Genetically engineered mouse models of brain cancer and the promise of preclinical testing. Brain Pathol 19: 132-143, 2009.

11. Lee LM, Seftor EA, Bonde G, Cornell RA and Hendrix MJ: The fate of human malignant melanoma cells transplanted into zebrafish embryos: assessment of migration and cell division in the absence of tumor formation. Dev Dyn 233: 1560-1570, 2005.

12. Stoletov K, Montel V, Lester RD, Gonias SL and Klemke R: High-resolution imaging of the dynamic tumor cell vascular interface in transparent zebrafish. Proc Natl Acad Sci USA 104: 17406-17411, 2007.

13. Jesuthasan S: Genetics and development. Zebrafish in the spotlight. Science 297: 1484-1485, 2002.

14. Stoletov K and Klemke R: Catch of the day: zebrafish as a human cancer model. Oncogene 27: 4509-4520, 2008.

15. White RM, Sessa A, Burke C, et al: Transparent adult zebrafish as a tool for in vivo transplantation analysis. Cell Stem Cell 2: 183-189, 2008.

16. Cross LM, Cook MA, Lin S, Chen JN and Rubinstein AL: Rapid analysis of angiogenesis drugs in a live fluorescent zebrafish assay. Arterioscler Thromb Vasc Biol 23: 911-912, 2003.

17. Westerfield M: The Zebrafish Book. A Guide for the Laboratory Use of Zebrafish (Danio rerio). Eugene, OR, University of Oregon Press, 2007.

18. Nicoli S, Ribatti D, Cotelli F and Presta M: Mammalian tumor xenografts induce neovascularization in zebrafish embryos. Cancer Res 67: 2927-2931, 2007.

19. Nicoli S and Presta M: The zebrafish/tumor xenograft angiogenesis assay. Nat Protoc 2: 2918-2923, 2007.

20. Serbedzija GN, Flynn E and Willett CE: Zebrafish angiogenesis: a new model for drug screening. Angiogenesis 3: 353-359, 1999.

21. Thisse $C$ and Thisse B: High-resolution in situ hybridization to whole-mount zebrafish embryos. Nat Protoc 3: 59-69, 2008.

22. Adhikary S and Eilers M: Transcriptional regulation and transformation by Myc proteins. Nat Rev Mol Cell Biol 6: 635-645, 2005.

23. Spitsbergen JM, Tsai HW, Reddy A, et al: Neoplasia in zebrafish (danio rerio) treated with 7,12-dimethylbenz[a]anthracene by two exposure routes at different developmental stages. Toxicol Pathol 28: 705-715, 2000.

24. Spitsbergen JM, Tsai HW, Reddy A, et al: Neoplasia in zebrafish (Danio rerio) treated with N-methyl-N'-nitro-N-nitrosoguanidine by three exposure routes at different developmental stages. Toxicol Pathol 28: 716-725, 2000.

25. Shepard JL, Amatruda JF, Stern HM, et al: A zebrafish bmyb mutation causes genome instability and increased cancer susceptibility. Proc Natl Acad Sci USA 102: 13194-13199, 2005.

26. Amatruda JF, Shepard JL, Stern HM and Zon LI: Zebrafish as a cancer model system. Cancer Cell 1: 229-231, 2002.

27. Mione MC and Trede NS: The zebrafish as a model for cancer. Dis Model Mech 3: 517-523, 2010.

28. Chi A, Norden AD and Wen PY: Inhibition of angiogenesis and invasion in malignant gliomas. Expert Rev Anticancer Ther 7: 1537-1560, 2007. 
29. Leung DW, Cachianes G, Kuang WJ, Goeddel DV and Ferrara N: Vascular endothelial growth factor is a secreted angiogenic mitogen. Science 246: 1306-1309, 1989.

30. Plate KH, Breier G, Weich HA and Risau W: Vascular endothelial growth factor is a potential tumour angiogenesis factor in human gliomas in vivo. Nature 359: 845-848, 1992.

31. Roy H, Bhardwaj S and Yla-Herttuala S: Biology of vascular endothelial growth factors. FEBS Lett 580: 2879-2887, 2006

32. Neufeld G, Tessler S, Gitay-Goren H, Cohen T and Levi BZ: Vascular endothelial growth factor and its receptors. Prog Growth Factor Res 5: 89-97, 1994.
33. Neufeld G, Cohen T, Gengrinovitch S and Poltorak Z: Vascular endothelial growth factor (VEGF) and its receptors. FASEB J 13: 9-22, 1999.

34. Habeck H, Odenthal J, Walderich B, Maischein H and SchulteMerker S: Analysis of a zebrafish VEGF receptor mutant reveals specific disruption of angiogenesis. Curr Biol 12: 1405-1412, 2002.

35. Liang D, Xu X, Chin AJ, et al: Cloning and characterization of vascular endothelial growth factor (VEGF) from zebrafish, Danio rerio. Biochim Biophys Acta 1397: 14-20, 1998. 